\title{
Institutional environment for the development of the sharing economy
}

\author{
Nataliia Simchenko ${ }^{1, *}$, and Stas Nagornyi ${ }^{2}$ \\ ${ }^{1}$ Maykop State Technological University, Maykop, Russia \\ ${ }^{2}$ V.I. Vernadsky Crimean Federal University, Simferopol, Russia.
}

\begin{abstract}
The article is devoted to studying prerequisites for the formation of the institutional environment of the economy of joint consumption in the current digital environment. From the standpoint of institutional theory, the institutional environment is understood as an ordered set of institutions that determine the constraints for economic agents in making innovative managerial decisions. The peculiarities of the institutional environment functioning are determined by the mutual support of the institutions, which is ensured by performing their respective functions. It is proved that the development of the relationship between formal and informal institutions is one of the key factors of institutional dynamics. It needs not only the formation but also the perception of the rules and norms accepted in society. Formal constraints, rules, and institutions emerge for the most part from the principles of already existing informal rules and mechanisms that enforce them. The functions of the institutional environment, which generally represent the activities aimed at streamlining the actions to maintain stability in society, are highlighted.
\end{abstract}

\section{Introduction}

The scientific significance of the institutional environment of joint consumption economy development study is due to the development of fundamental principles and conceptual provisions of the theory of joint consumption economy in the digital environment based on the integration of scientific achievements in the field of institutional theory, sustainable development paradigm, digital economy paradigm, entrepreneurship theory, platform economy theory, smart cities concept, and formation of a new pioneering theory on this basis, providing, inter alia, for the development of a new pioneering theory, which includes the development of a closed-loop economy based on AI engineering, Digital Twins, Internet of Behaviors, Hyperautomation, and other breakthrough digital technologies.

The relevance of the mentioned scientific problem is connected with the need to solve the aggravating social and resource-ecological problems, growth of intellectualization, and dematerialization of technologies in the digital environment. The unprecedented development of digital technologies is fundamentally changing the concept of sustainable development of society, introducing major shifts in the structure of economic systems, which leads to the need to rethink opportunities for economic growth and consumption of raw

* Corresponding author: natalysimchenko@yandex.ru 
materials and energy resources. The development of a co-consumption economy requires comprehensive social, institutional, technological, and cultural changes in a linear economywide mechanism. The growing contradictions between the increasing level of digital technology development and the loss of sustainability of economic systems require the platform introduction of new business models, stimulating the development of new forms of technological entrepreneurship based on breakthrough information technologies, which will create new jobs, including in the sphere of waste-free production. Thus, the relevance of solving the scientific problem is due to the theoretical and methodological foundations of digital economy development, the applied nature, and the needs of modern society to ensure sustainable economic development on the circular economy principles and the transformation of technological entrepreneurship models.

\section{Study methodology}

The scope of the study task is determined by the synthesis of scientific schools in the field of institutional theory, sustainable development paradigm, digital economy paradigm, entrepreneurship theory, platform economy theory, the concept of smart cities, and the formation of a new pioneering theory on this basis, providing, inter alia, the development of a closed-loop economy based on AI engineering, Digital Twins, Internet of Behaviors, Hyperautomation technologies.

The complexity of the specific task of the project consists of applying an integrative approach to solving the problem of formation of the institutional environment for the development of the economy of joint consumption.

\section{Results of the study}

The development of the sharing economy under the influence of Industry 4.0 is a leading trend in the development of the economic system. This leads to the transformation of social and economic institutions. The development of institutions leads to the reduction of transaction costs, which is undoubtedly a significant effect of the development of the digital economy.

The scarcity of resources changes the attitude to their consumption culture, which led to the transition from a consumer society to the sharing economy, characterized by the joint (collective) use of goods and services. These trends nowadays are manifested in different spheres (for example, carsharing in its various manifestations is developing in the sphere of transport infrastructure; movable things sharing also gain popularity in the sphere of using real estate, etc.).

Most modern publications consider the economics of collaborative consumption from the perspective of the development of digital platforms and digital services $[1,2,3,6,8]$. Several works are devoted to assessing the impact of the joint consumption economy development on the sustainability of modern society $[4,7,8]$.

The contradictory nature of the sharing economy phenomenon is obvious. As emphasized in [9], the sharing economy is based on three postulates: 1) access economy, 2) platform economy and 3) community economy. When presenting these three components of the economy of joint consumption, the authors emphasize, on the one hand, their inseparability and unity, and, on the other hand, their contradictory nature $[9,10]$.

The importance of the institutional environment for the development of the coconsumption economy cannot be overemphasized. Traditionally, the institutional environment is considered a set of institutions affecting the structuring of social relations 
between economic agents. The institutional environment has some properties, among which it is worth highlighting, such as integrity, stability, hierarchy, heterogeneity.

The institutional environment will be understood as an ordered set of institutions that determine the constraints for economic agents in making innovative management decisions. The peculiarities of the institutional environment functioning are determined by the mutual support of the institutions, which is ensured by performing their respective functions.

The functions of institutions are generally activities that contribute to the maintenance of stability in society. Along with this, dysfunction is the activity of institutions that makes it impossible to meet the needs of the innovation system and leads to its destruction. Dysfunction occurs if its orientation characterizes the activity of an innovative institution not towards society but self-sufficiency. The totality of social functions of different institutions forms the common functions.

According to the classification of T. Parsons, social institutions have the following functions [11]: adaptation to the environment; ensuring the implementation of the system's main objectives and mobilization of means for their implementation; coordination and integration of activities of the system's structural elements; maintaining the values dominating in the society.

Let us note that the development of the relationship between formal and informal institutions is one of the key factors of institutional dynamics. It needs not only the formation but also the perception of the rules and norms accepted in society. Formal constraints, rules, and institutions emerge for the most part from the principles of already existing informal rules and mechanisms that enforce them.

Based on the analysis of scientific literature [12-13], we identify the following functions of the institutional environment:

1. Cognitive function. Institutions ensure the existence of processes and phenomena occurring in the economic life of society, form a system of economic views of society.

2. Practical function. It is manifested in the definition of theoretical conclusions and analysis of their reliability through economic activity, search for effective forms and ways of managing economic processes, and creating the adequate structure of management at all economic levels.

3. Integrating function. Economic institutions contribute to the realization of subjects as individuals of social production, facilitate establishing economic contacts, provide savings due to transaction costs. The reduction of uncertainty in the external system provided by the existence of institutions allows planning and resorting to long-term investments, creating more value. The money saved by researching and predicting the behavior of actors can be used for economic purposes. In an uncertain environment, in the absence of functioning institutions, economic counterparties not only face a reduced expected return on future investments but are also forced to spend additional funds on precautionary measures in economic activities, such as insurance.

From the integrating function follows the next one, which is one of the main ones reduction of transaction costs. The theory of transaction costs appeared in R. Coase's article "The Nature of the Firm", now recognized as a classic. R. Coase began his research with an analysis of the two, according to him, key institutions that are most essential for economic theory - the firm and the market, characterizing the institutional structure of the economic system. He introduces a new category into economic analysis - "transaction costs" [12]. P. Coase answers the question of the reason for merging into a firm. It is known that the market provides freedom, and the firm restricts it accordingly. To be successful in the marketplace, an entrepreneur must have reliable information, which requires significant transaction costs. Consequently, transaction costs are central to the analysis of economic institutions.

Institutions are a means of reducing transaction costs. Economic actors seek to reduce their own costs and increase profits. The main idea of reducing transaction costs is to reduce 
uncertainty in the economic system and respect property rights through stable rules of conduct. These rules of conduct are the institutions. As a result of the high degree of institutional coherence, transaction costs will be minimized. When transaction costs are rising, various non-standard models of economic institutions, which contribute to reducing transaction costs, are purposefully created to reduce them. This approach to the use of transactional efficiency of institutions explains the low efficiency of the use in a particular country of economic institutions that have proved positive in other states.

4. Information function. It consists of the accumulation, selection, and transmission of information in space and time. Performing the informational function, economic institutions ensure the continuity of society's reproduction. The counterparties' awareness of the content of the institution regulating behavior is a condition for the coordination of economic activities of the subjects. If one of the counterparties knows how to behave in certain circumstances and the other does not, coordination will be disrupted, resulting in unproductive costs to the parties involved. Efficiently operating institutions raise the awareness of transaction participants about the state of the market and economy as a whole, thus minimizing the costs of information surfing and streamlining the activities of economic agents.

5. Regulatory function. This function consists in the fact that economic institutions direct the activities of economic individuals in the direction that is most beneficial to the economy as a whole and try to slow down or stop at all the activities of actors that bring negative consequences. Institutions as the basis of norms and rules in society create the appearance of security and confidence of the individual to assure his rights and freedoms. As a result, people's material and intellectual resources are used to their best advantage.

Within the regulatory framework, the coordinating and distributive functions can be noted. The coordinating function manifests itself in reduced access to resources and a variety of ways of using them. By restricting possible variants of actions and directions of behavior or even prescribing only one admissible way of behavior, institutions also regulate behavior of economic agents, which find themselves in a situation characterised by conditions of application of an appropriate valid norm. An analysis of the institution's content in a particular situation gives each of the economic actors the knowledge of how the counterparty should behave. On this basis, individuals can and are likely to apply their own line of conduct, taking into account the expected actions of the opposing party, which means applying coordination in their actions.

6. Cultural function. The cultural context and institutional environment create a set of possible organizational alternatives and thereby determine the structure and size of transaction costs. In contrast, organizational alternatives differ among themselves in the types of transactions concerning which they act as the most appropriate management mechanisms. Institutions order the relations between people and discard the forms of behavior that would lead to the disintegration, and consequently the death, of human society. Thus, economic institutions harmonize and coordinate the economic activities of the participants in society.

The structure of economic interaction between economic subjects is streamlined, which allows them to predict the consequences of their actions. According to D. North, one of the leading representatives of institutional theory, "the main role that institutions play in society is to reduce uncertainty by establishing a stable (though not necessarily effective) structure of interaction between people. Any institution, reducing many valid ways of action, affects the redistribution of resources by economic subjects, performing a distributive function. The allocation of resources, costs, and benefits is affected not only by the rules and regulations whose content is the transfer of goods and benefits from one counterpart to another but also by those not directly related to these issues [13].

7. Non-entropic (system-forming) function. It manifests itself in ensuring stability, maximizing the level of organization of the economy of an individual state, the ability to level out, to a certain extent, the resulting fluctuations in changes. The implementation of this 
function becomes possible since the relationship between the subject and institutions is characterized by a certain and at the same time essential inequality: carrying out his activity, generated by needs, through an institution, an individual is forced to adapt the structure of his needs and interests or methods of their satisfaction to institutional norms, while the influence of an individual subject on institutions is negligible. This explains the ability of institutions to perform a systemic function.

8. Complementarity function. Institutions complement each other, and the desired economic effect is achieved through close interrelated institutional changes. In a developed economic system, economic relations are also characteristic of contacts between institutions since the development of the economy is accompanied by a struggle between them. Institutions act as a mechanism for coordinating the actions of economic individuals who lack market coordination. The latter runs up against the boundaries of the time period over which the cost-benefit comparison takes place.

9. Educational function. The educational effect of the institute is aimed at a separate sector of public consciousness - the legal consciousness, the goals of such an impact are also established. The educational effect of the institute includes an ideological aspect, implemented mainly employing social propaganda and aimed at improving public culture. Rules of social behavior are transmitted through the educational function of the institute, its values, as an element of the spiritual culture of society, and skills.

Each of the above socio-economic functions of institutions has its own regulatory mechanism through which the institution implements its economic goals and objectives.

Note that the development of the economy of joint consumption leads to the transformation of social institutions that can both accelerate and hinder the development of the economic system. The importance of the institutional environment for the development of the sharing economy can hardly be overestimated. Traditionally, the institutional environment is considered a set of institutions affecting the structuring of social relations between economic agents. Institutional environment is a complex ordered system of basic and infrastructural economic institutions that create conditions for social reproduction through the formation of generally binding rules and mechanisms of their maintenance, on the other hand - the system of relations between economic subjects and institutions regarding the satisfaction of economic subjects' needs for institutions and institutional mechanisms. The institutional environment has some properties, among which it is worth highlighting, such as integrity, stability, hierarchy, heterogeneity.

Thus, by characterizing the basic regulatory relations between economic agents, institutions change the nature of joint use of property rights. Undoubtedly, the prospect of further research is the analysis of networks and networking in the development of institutions supporting the collaborative consumption economy in the context of digital transformation of the economy.

\section{References}

1. I. Gelishanov, T. Judina, A. Babkin, St. Petersburg State Polytechnical University Journal, Economics 11(6), 22 (2018). DOI: 10.18721/JE.11602

2. R. Gupta, C. Mejia, Y. Kajikawa, Technological Forecasting and Social Change 147, 100 (2019).

3. V. Kupriyanovsky, D. Namiot, S. Sinyagov, Int. J. of Open Information Technologies 11, 59 (2016).

4. B. Nikitina, M. Korsun, I. Sarbaeva, V. Zvonovsky, Intelligent Systems and Computing 908, 376 (2020).

5. D. North, World Development 17(9), 1319 (1989).

6. T. Lin, Advances in Intelligent Systems and Computing 965, 329 (2020). 
7. Z. Mi, D. Coffman, Nature Communications 10(1), 1214 (2019). doi: 10.1038/s41467019-09260-4.

8. O. Panfilova, V. Okrepilov, S. Kuzmina, MATEC Web of Conferences, 01032 (2018).

9. A. Acquier, T. Daudigeos, J. Pinkse, Technological Forecasting and Social Change 125, 1 (2017). doi: 10.1016/j.techfore.2017.07.006.

10. D. Arcidiacono, A. Gandini, I. Pais, Sociological Review 66(2), 275 (2018). doi: 10.13169/workorgalaboglob.11.2.0039

11. T. Parsons, N. Smelser, Economy and Society: A Study of the Integration of Economic and Social Theory (N.Y., 1965)

12. R. Coase, Economica: Blackwell Publishing 4(16), 386 (1937)

13. D. North. Institutions, institutional change and economic performance, 107 (Cambridge, 1990) 